\title{
2 nOTHCE
}

CoRTions of THIS REpORT ARE ILLEIBLE.
has been reproduced from the best avaibite
cody to permit the brosdest possible avail

silin.

\section{IMPLICIT FUNCTION METHOD \\ FOR THE LIOUVILLE EQUATION*}

\author{
S. KHEIFETS \\ Stanford Linear Aecelerator Center \\ Slanford University, Stanford, California 04805
}

A number of problems may be named for which the solution of the corresponding Liouville equation might be useful. For example the interaction of an electron flow with an ungridded gap may be approached from this direction. Another example is obtaining the distribution function of a particle bunch at the exit of a transport line containing nonlinear elements should the distribution functions at the entrance is known. List of such problems may be extended.

In the present short note I derive a rather general method of solving the Liouviile equation. No discussion will be presented on how fast the solution can be found or how convenient the method is in practical spplications. Nor any numerical calculations will be done. All these tasks can be subjects of further studies sbould the method become useful later.

Fo: the sake of simplicity and to shorten derivation I start from the case of a one-dimensional nonrelativistic motion. In addition 1 assume that the force acting in a particle does not depend on its velocity. Next section contains the results for general 3-D case and the force depending on velocity as wcll.

- Work supported by the Department of Dnergy, contract DE-AC03-76SF00515 


\section{One-Dimendonal Motlon}

Let us assume the following equations of a particle motion

$$
\begin{aligned}
& \frac{d x}{d t}=v \\
& \frac{d v}{d t}=F(x, t)
\end{aligned}
$$

\subsection{The LIOUVILLE Equation}

Io the phise space $(x, v)$ the particle mation may be equivalently described by the corresponding Liouville equation for the distribution function $\psi=\psi(x, u, t)$ :

$$
\left\llcorner\psi \equiv \frac{\partial \psi}{\partial t}+v \frac{\partial \psi}{\partial x}+F(x, l) \frac{\partial \psi}{\partial v}=0\right.
$$

Note that unlike (1.1) and (1.2) where $z=x(t)$ and $v=v(t)$, in Eq. (1.3) $x$ and $v$ are independent variables and they do not depend on $l$.

\subsection{The Characteristic Function}

The solution of (1.3) may be found in terms of a certain characteristic function $X\left(x, v, t, t^{\prime}\right)$ of two time variables $t$ and $t^{t}$ which is defined implicitly by the followiag equation:

$$
G \equiv X-x+v \cdot\left(t-t^{\prime}\right)-\int_{t^{\prime}}^{t}\left(t^{\prime \prime}-t^{\prime}\right) F\left(X, t^{\prime \prime}\right) d t^{\prime \prime}=0
$$

Given the function $F(x, t)$, the characteristic function $X\left(x, v, t, t^{\prime}\right)$ ean in principle be found. I will assume in what follows that $X\left(x, v, t, t^{\prime}\right)$ is known.

From the definition (1.4) inmediately follows that at $t^{\prime}=t$

$$
\boldsymbol{X}(\boldsymbol{x}, \mathbf{x} \cdot \cdot \boldsymbol{x}
$$

Reminknpln $n$, erty of $X\left(x, v, t, t^{\prime}\right)$ is that ie satisfies the Liouville equation (1.3) f(u, wist time variable and for any value of the second variable $t^{\prime}$. To see that 
calculate $L G=0$ (since $G=0$ ):

$$
L G=L X-\int_{t^{\prime}}^{t} d t^{n} \cdot\left(t^{n}-t^{\prime}\right) \cdot \frac{\partial F}{\partial X} \cdot L X=0
$$

Let us denote for the time being

$$
\begin{aligned}
C X & \equiv f\left(x, v, t, t^{\prime}\right) \\
\left(t^{\prime}-t\right) \cdot \frac{\partial F}{\partial x} & \equiv g\left(x, v, t, t^{\prime}\right)
\end{aligned}
$$

Then for $f$ we have the integral equation

$$
f(x, t, t, \tau)=\int_{t}^{t} d t^{\prime} d\left(x, v, t, t^{\prime}\right) f\left(x, v, t, t^{\prime}\right)
$$

Under certain conditions (1.9) has only the trivial solution $f(x, v, t, \tau)=0$ for any $r$. One notes first of all that $f(x, y, t, t)=0$.

Expand now all the functions in (1.9) in the Taylor series in $u=t-r$ and perform integration:

$$
-f_{0}^{A}-u+\frac{f_{0}^{\prime \prime}}{2} u^{2}-\ldots=g_{0} f_{0}^{t} \frac{u^{2}}{2}-\frac{g_{0} f_{0}^{\prime \prime}}{6} u^{3}-\frac{g_{0} f_{0}}{3} u^{3}+\ldots
$$

All quantities with the subscript 0 here denote the value of corresponding function at $u=0$ (or $r=t$ ).

Comparing now terms with equal powers in $u$ one finds $f_{6}=0, f_{0}^{k}=0$ and so on. Any function $f$ which allows the Taylor expansion and bas all its derivatives zero is zero itself. This means that $\boldsymbol{X}$ indeed satisfies the Liouville equation unless the function $F(x, t)$ has no Taylor expansion. We will not consider special cases like that.

\subsection{Solution Of The Llouville Equation}

Suppose now that at the beginning of the system $x=0$ the distribution function $\psi$ is known

$$
\left.\psi(x, v, t)\right|_{x=0}=\psi_{0}(v, t)
$$


I will show that st ayy other place $x$ of the syotem, the wolntion of (i..8) which atisfies the bonndary condition (1.10) can be written as follows:"

$$
\psi(x, v, l)=\downarrow_{0}(V(x, v, t), \theta(x, v, t)),
$$

where

$$
V(x, v, t)=v-\int_{\Theta(z, v, t)}^{t} F\left(X\left(x, v, \ell, t^{\prime}\right), t^{\prime}\right) d t^{\prime}
$$

and $\Theta(x, v, t)$ is a such a function implicity defined by the equation:

$$
F \equiv x-v \cdot(t-\theta)+\int_{\theta}^{t}\left(t^{\prime}-\theta\right) \cdot F\left(X\left(x, v, t, t^{\prime}\right), t^{\prime}\right) d t^{\prime}=0
$$

which goes into $i$ for $x=0$ :

$$
\left.\theta(x, v, n)\right|_{z=0}=1
$$

In (1.12) and (1.13) the function $X\left(x, v, l, t^{\prime}\right)$ is the one defined in (1.4). To prove that (1.11) indeed satisfies (1.3) one first noles that

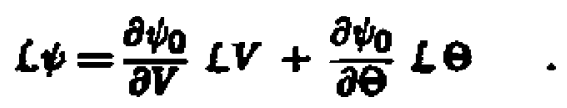

Next find $\boldsymbol{L}=\mathbf{0}$ :

$$
L F=-\int_{\theta}^{t} d t^{\prime} F\left(X, t^{\prime}\right) \cdot L \theta=0
$$

Since (1.15) should be true for any value of $x, v$ and $t$, it follows from (1.15)

$$
L \theta=0
$$

The calculation of $L V$ gives

$$
L V=F(X, \theta) \cdot L \theta=0
$$

and hence $L \psi=0$.

Using (1.14) one finds $\left.V(x, v, t)\right|_{x=0}=v$ and bence (1.10) is satisfied also. 


\section{General Croe}

Suppose now that the motion of a particle cas be described by equations:

$$
\begin{aligned}
& \frac{d \vec{P}}{d t}=\vec{v} \\
& \frac{d \vec{v}}{d t}=\tilde{F}(\vec{P}, \vec{v}, t)
\end{aligned}
$$

The Liouville equation for the distribution function $\psi=\psi(\vec{f}, \vec{\nabla}, t)$ in B-D phase space is:

$$
L \psi \equiv \frac{\partial \psi}{\partial l}+\nabla \cdot \nabla_{\nabla} \psi+\vec{F} \nabla_{\nabla} \psi=0
$$

To solve this equation by the same method one needs now two vector chasacteristic functions:

$$
\begin{aligned}
& \vec{P} \equiv \vec{U}-\vec{\nabla}+\int_{i}^{\ell} d t^{\prime \prime} \vec{F}\left(\vec{S}\left(\vec{r}, \vec{v}, t, t^{\prime \prime}\right), \vec{D}\left(\vec{F}, \vec{v}, \ell, t^{\prime \prime}\right)\right) d t^{\prime \prime}=0 \\
& \vec{G} \equiv \vec{S}-\vec{F}+\vec{v} \cdot\left(t-t^{\prime}\right)-\int_{t^{\prime}}^{t} d t^{n}\left(t^{n}-t^{\prime}\right) \vec{F}(\vec{S}, \vec{V}) d t^{n}=0
\end{aligned}
$$

Functions $\vec{S}$ and $\vec{U}$ bave the following propertiea:

$$
\begin{aligned}
\vec{S}(\vec{\nabla}, \vec{v}, t, t) & =\vec{\nabla} \\
\vec{U}(\vec{\nabla}, \vec{\nabla}, t, t) & =\vec{\nabla} \\
\iota \vec{S} & =0 \\
\iota \vec{U} & =0
\end{aligned}
$$

The proof of these properties is a multi-dimensional generalization of the proof given in Section 1.

Solution of the Liouville equation can be expressed in terms of the charasteristic functions $\vec{S}$ and $\vec{U}$. 


\subsection{The Initial Value Problem}

If $\psi$ is known at $t=t_{0}$ :

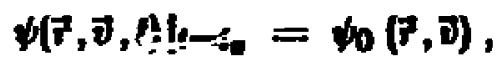

then for any subsequent time moment:

$$
\phi(\vec{\nabla}, \vec{\nabla}, \boldsymbol{l})=\boldsymbol{\psi}_{0}(\overrightarrow{\boldsymbol{R}}, \vec{\nabla})
$$

where

$$
\begin{gathered}
\vec{\nabla}=\vec{\nabla}-\int_{t_{0}}^{t} d t^{\prime} \vec{F}\left(\vec{\xi}, \bar{D}, t^{\prime}\right), \\
\vec{R}=\vec{r}-\vec{v} \cdot\left(t-t_{0}\right)+\int_{t_{0}}^{t} d t^{\prime} \cdot\left(t^{\prime}-t_{0}\right) \cdot \vec{F}\left(\vec{S}, \vec{D}, t^{\prime}\right),
\end{gathered}
$$

where $\vec{S}$ and $\vec{D}$ are implicitly defined in (2.4) and (2.5).

2.2 The BOUNDARY VAlue Problem

If $\psi$ is known at some point $\vec{z}=0$

$$
\left.\psi(\vec{F}, \vec{v}, t)\right|_{F=0}=\psi_{0}(\vec{t}, t),
$$

then at any other place:

$$
\psi=\psi_{0}(\vec{V}, \theta)
$$

where

$$
\vec{V}=\vec{\nabla}-\int_{\theta}^{t} d t^{\prime} \vec{F}\left(\vec{S}, \vec{U}, t^{\prime}\right)
$$

and function $\theta$ is impticity defined as such a solution of the equation

$$
\vec{F}-\vec{\theta} \cdot(t-\theta)+\int_{\theta}^{t} d l^{\prime} \cdot\left(t^{\prime}-\theta\right) \vec{F}\left(\vec{\xi}, \vec{D}, t^{\prime}\right)=0
$$

which satisfies the condition:

$$
\left.\theta(\vec{r}, \vec{v}, t)\right|_{p=0}=t
$$




\section{Concluslon}

The method of solving the Liouville equation described here rewts heavily on the existence of two implicit functions $\vec{U}$ and $\vec{S}$. Provided these functions are known many interesting problems can be solved by means of integration. For example, any bunch characteristics at the exit of a system (rms values, invariant curves, Poincaré sections of different kind, etc.) can be found from the known distribution function.

The computation of the characteristic functions $\vec{V}$ and $\vec{S}$ is transformed hcse to a (nonlinear) integral equation. There are no known methods of solving such equations other than a numerical one. At present time one can only guess which one of numerical solutions - direct solution of the differential equation (2.3) or solution of an integral equation - is faster and less expensive. The only general elue to this question is that there are cases when an integration is stabje numerically while a differentiation is unstable.

\section{Acknowledgement}

I am grateful to A. Chao, Ph. Morton and R. Ruth tor belpful discussion and in addition to $\mathrm{A}$. Chao for a number of comments be made while readiug the manuscript.

\section{DISCLAIMER}

This report was prepared as an account of work sponsored by an agency of the United States Government. Nolther the United States Government nor any agency thereol, nor any of their employees, makes any warranty, express or implied, or assumes any legal liability or responsibility for the accuracy, completeness, or usefulness of any information, apparatus, product, or process disclased, or represents that its use would not infringe privately owned rights, Reference herein to any specific commercial product, process, or service by trade name, trademark, manufacturer, of otherwise does not necessarily constitute or imply its endorsement, recommendation, or faroring by the United States Government of any agency tbereof. The views and opinions of authors expressed herein do not necessarily state of reflect those of the United States Government of any agency thereof. 